\title{
Repenser le modèle de développement agricole du Maroc pour l'ère post Covid-19
}

\author{
Mohamed Taher Sraïri* \\ Institut Agronomique et Vétérinaire Hasan II, Rabat, Maroc
}

\begin{abstract}
Résumé - Le secteur agricole constitue une activité importante au Maroc, représentant $14 \%$ du produit national brut et employant $40 \%$ de la population active. Avec l'émergence de la pandémie de la Covid-19, il a été ébranlé par des incertitudes économiques et par la distanciation physique qui a accentué les contraintes du travail. Toutefois, l'agriculture s'est imposée comme une activité cruciale, puisqu'elle a continué à assurer l'approvisionnement alimentaire à des prix abordables. La pandémie, qui s'est déclarée en une année très sèche, a offert une opportunité pour réviser les principales contraintes auxquelles fait face le secteur agricole : manque d'eau et faible rémunération du travail. Elle a aussi attiré l'attention de l'opinion publique sur les quantités importantes de denrées vivrières importées. La balance des paiements des produits alimentaires demeure négative, malgré des exportations de produits agricoles mises en relief par le discours officiel. En outre, des études récentes ont montré que ces dernières dépendent avant tout d'usages accrus et souvent déraisonnables d'eau souterraine, malgré les subventions à la conversion des systèmes d'irrigation gravitaire vers le goutte-à-goutte. Des suivis d'exploitations ont démontré que ces subventions ont généré une expansion de la surface irriguée avec des cultures à besoins hydriques élevés. Cette situation doit être considérée comme l'opposé de l'objectif recherché : une amélioration de la valorisation de l'eau. Il faut donc reconnaitre que l'agriculture de l'ère post Covid-19 devra être différente. Un changement de paradigme doit s'opérer où la valorisation de l'eau pluviale figurera dans les priorités, de concert avec la souveraineté alimentaire. Il doit englober différentes dimensions, comme la préservation de l'environnement, le développement du monde rural et de systèmes alimentaires durables, mais aussi garantir l'inclusion sociale et de meilleures rémunérations du travail agricole, afin d'assurer son attractivité et réduire l'exode rural.
\end{abstract}

Mots clés : Agriculture / Maroc / souveraineté alimentaire / travail / valorisation de l'eau

\begin{abstract}
Rethinking the agricultural development model in Morocco in the post Covid-19 era. The agricultural sector is strategic for the Moroccan economy, still accounting for around $14 \%$ of the Gross Domestic Product and employing 40\% of the active population. With the emergence of the Covid-19 pandemic, it has been literally shaken up, with growing uncertainties with regard to market opportunities and physical distancing which has amplified work constraints. However, citizens have rapidly acknowledged the importance of agriculture, as it ensured a regular supply of food during the lockdown at relatively affordable prices. The pandemic, which has emerged in a particularly dry year, has provided an opportunity to revise the constraints facing the agricultural sector, particularly water scarcity and limited work remuneration. It has also shown to consumers the significant share of imported staple food. In fact, the food trade balance remains in deficit despite the exports of high value commodities, which are emphasized by the public authorities. Moreover, recent studies have shown that these exports rely on increasing amounts of groundwater uptakes. This has happened despite the significant subsidies awarded to farmers to convert gravity irrigation to drip irrigation systems. On-farm investigations have demonstrated that subsidies mainly resulted in an expansion of the area with cash crops needing significant amounts of water. This situation is the opposite of the goal sought by the agricultural policy, i.e. a higher economic water productivity. It is therefore crucial to recognize that the post Covid-19 agriculture should be different. Indeed, there is a need for a paradigm shift where rain-fed agriculture has to get at the top of the political agenda, with more attention to food sovereignty. This has to encompass wide topics, such as environment
\end{abstract}

\footnotetext{
$\overline{\text { *Auteur de correspondance }}$ : mt.srairi@iav.ac.ma
} 
preservation, rural development and sustainable food systems, to ensure social inclusiveness, and guarantee better wages that can increase the attractiveness of work within farming activities and reduce rural exodus.

Keywords: Agriculture / food sovereignty / Morocco / water productivity / work

\section{Introduction}

On ne résout pas un problème avec les modes de pensée qui l'on engendré Albert Einstein (1879-1955)

Au Maroc, depuis l'Indépendance (1956), l'agriculture a toujours été considérée comme une priorité, au vu du poids des affaires rurales dans les questions de gouvernance (Leveau, 1972). L'amélioration des performances agricoles a été un objectif constant de l'État, surtout par le développement de l'irrigation. Ce choix stratégique était induit par le climat aride à semi-aride qui règne sur le pays et, depuis les années 1980, par la plus grande fréquence des sécheresses (Barakate et Handoufe, 1998).

En 2008, l'État marocain a lancé une ambitieuse stratégie, le «Plan Maroc Vert» (PMV) qui, entre autres, visait l'extension des surfaces irriguées et l'économie de l'eau par la promotion de l'irrigation localisée, en subventionnant à des niveaux de 80 et même $100 \%$ (dans les exploitations de moins de $5 \mathrm{ha}$ ) les investissements nécessaires : creusage de puits et forages, équipements d'irrigation (pompes, bassins, tuyaux, goutteurs, etc.). Le PMV a en même temps encouragé des entrepreneurs à accéder à des terres du domaine public, par des baux de longue durée, assimilés par certains à de l'accaparement foncier (Mahdi, 2014). Ces mesures ont indéniablement débouché sur de nouvelles dynamiques agricoles, grâce la mobilisation de fonds importants (environ 10 milliards d'euros en 10 ans). Le PMV a permis un accroissement des productions de fruits, de légumes, d'huile d'olive et des viandes blanches, mais il n'a pas pu assurer une augmentation notable de la production d'aliments de base comme les céréales, les grains de légumineuses, le lait, les huiles végétales, etc. De ce fait, le Maroc demeure dépendant d'importations massives pour ces produits (Tab. 1). Inversement, le PMV a favorisé certaines filières d'exportation (légumes primeurs, agrumes, huile d'olive) permettant ainsi au pays d'acquérir des devises. Cette stratégie a entièrement reposé sur la mondialisation des échanges commerciaux et leur fluidité. Toutefois, avec l'émergence de la pandémie de la Covid-19, certains pays exportateurs de céréales ont annoncé qu'ils pourraient adopter des mesures protectionnistes et réserver en priorité leur production aux besoins de leurs consommateurs (Espita et al., 2020). Par conséquent, plusieurs voix au Maroc ont alerté l'opinion publique sur les risques inhérents aux choix agricoles stratégiques du pays quant à sa sécurité alimentaire. Comme l'actuelle pandémie a débuté avec la fin du PMV, il apparaît important qu'un bilan de ce programme soit établi en tenant compte des effets négatifs et des opportunités observés durant le premier semestre de cette crise sanitaire. Ceci doit être réalisé en mobilisant les savoirs scientifiques pour orienter efficacement les choix politiques (Sager et al., 2020) et contribuer à l'instauration de débats sains qui reposent sur la diversité des avis: expertise, connaissances issues du terrain, etc. Divers auteurs considèrent en effet que le PMV a éludé les risques avérés de l'intensification agricole en conditions arides et semi-arides, tout comme il a mésestimé la diversité des structures et des stratégies des exploitations agricoles (Akesbi, 2015; Faysse, 2015). Dans ce contexte, cet article propose une analyse des voies possibles que pourrait emprunter l'agriculture marocaine dans l'ère post Covid-19 afin qu'elle réponde mieux à l'objectif de sécurité alimentaire. Pour ce faire, sur la base d'une bibliographie sélective, une analyse des impacts les plus marquants du PMV (aussi bien sur les exploitations agricoles que sur différentes filières) sera effectuée, à partir par les données relatives aux productions et aux échanges de produits agricoles et alimentaires du Maroc (Office des Changes, 2020; ONICL, 2020).

\section{Impacts de la Covid-19 sur l'économie et l'emploi agricoles au Maroc}

La pandémie de la Covid-19 a eu un impact catastrophique sur l'économie marocaine en 2020, engendrant une récession marquée, la première depuis 1995, avec une baisse de plus de $6 \%$ du Produit national brut (PNB) et un déficit budgétaire de l'État de $8 \%$ (World Bank, 2020). Les effets multiples déjà constatés de cette crise (économiques, financiers, sanitaires ...) et les impacts très négatifs qui vont s'ensuivre imposent d'emblée de réfléchir à des solutions innovantes, où le bienêtre humain doit avoir la primauté sur la rentabilité, remettant à l'agenda politique l'importance de systèmes éducatifs et sanitaires efficients et inclusifs, ainsi que de modes de production agricole et de consommation durables (Laborde et al., 2020). Ces idées doivent maintenant être investies au service de la conception de modèles qui permettent aux générations futures d'évoluer dans un environnement encore vivable, en dépit des défis immenses liés au changement climatique (Acuto, 2020).

Au début du confinement, en mars 2020, l'importance de l'agriculture s'est imposée à tous: les stocks de nourriture constitués en toute urgence par les ménages ont acquis une valeur inestimable, d'autant que des ruptures d'approvisionnement étaient redoutées. La population a pu aussi réaliser la contribution de l'agriculture à la fourniture d'emplois, à un moment où de nombreuses activités économiques se sont arrêtées en ville. De nombreux travailleurs ont été contraints de regagner leur village, et certains ont recouvré un revenu même modeste en s'employant temporairement dans l'agriculture. Ces observations convergent avec la thèse de l'anthropologue David Graeber (2018) qui insiste sur l'émergence d'emplois peu utiles surtout en milieu urbain, tandis que ceux qui s'activent dans les métiers nécessaires au bien-être de la population (l'enseignement, la santé, l'alimentation, etc.) jouissent de peu de considération. Ce constat s'applique avec justesse à l'agriculture, puisque ce secteur emploie au Maroc près de $40 \%$ de la population active mais ne génère que $14 \%$ 
Tableau 1. Importations de matières alimentaires au Maroc (2018 et 2019) $\left(\mathrm{x} 10^{6} €\right)$.

Table 1. Food imports in Morocco (2018 and 2019) (x 10 $€$ ).

\begin{tabular}{lcc}
\hline Matière première & 2019 & 2018 \\
\hline Blé & 785 & 776 \\
Tourteau oléagineux & 445 & 417 \\
Maïs & 445 & 395 \\
Sucre & 336 & 298 \\
Thé & 190 & 176 \\
Dattes & 138 & 97 \\
Fromages & 81 & 88 \\
\hline
\end{tabular}

Source: Adapté de Office des Changes (2020).

du PNB (Haut Commissariat au Plan, 2015). Cela correspond pour les actifs agricoles à des rémunérations réduites et instables, vu la saisonnalité des activités, surtout dans les systèmes de production pluviaux. De plus, les rapports de force dans les filières sont systématiquement au détriment des agriculteurs, ce qui limite leurs revenus et ceux de leurs employés. Même en zones irriguées, des études démontrent qu'un jour de travail agricole engendre un revenu souvent inférieur à 9 euros (Sraïri et al., 2018). Ceci est dû au volume de travail d'astreinte nécessité par l'élevage (affouragement et soins quotidiens) (Cournut et al., 2018) ainsi qu'à la difficile mécanisation des cultures (notamment le maraîchage). À cet égard, l'émergence de la pandémie a accentué ces difficultés économiques, les restrictions de déplacement ayant entravé le recours à la main-d'œuvre salariée et les possibilités de commercialisation des denrées périssables (fruits et légumes, particulièrement). De même, l'affaissement du pouvoir d'achat et la fermeture des lieux de restauration rapide a eu pour conséquence une baisse des prix de certaines denrées agricoles, notamment le poulet et les œufs, accentuant les déboires économiques de l'aviculture. Il convient aussi de rappeler que l'écrasante majorité des travailleurs agricoles ne jouissent d'aucune protection sociale (couverture des soins de santé et régime de retraite), ce qui ne les motive pas à rester dans ce secteur. Ceci explique les aspirations de nombreux ruraux, y compris les fils et filles d'agriculteurs, à l'exode rural ou à l'émigration pour chercher de meilleures conditions de vie (Bossenbroek et al., 2015). Ces dynamiques sont exacerbées par d'autres contraintes, notamment le manque d'eau.

\section{Le manque d'eau agricole et ses conséquences sur le secteur}

Avec une pluviométrie moyenne inférieure à $400 \mathrm{~mm} / \mathrm{an}$ sur plus de $85 \%$ de sa superficie, le Maroc souffre du manque d'eau agricole et ses productions végétales de stress hydrique (Fig. 1). La disponibilité en eau renouvelable a chuté à moins de $700 \mathrm{~m}^{3}$ par habitant et par an, alors qu'elle dépassait $2000 \mathrm{~m}^{3}$ dans les années 1960 (Fig. 2). Comme l'agriculture représente plus de $85 \%$ des usages hydriques, le manque d'eau constitue une menace avérée à son développement. Etudier la durabilité d'un modèle de développement agricole avant tout basé sur l'irrigation implique d'accorder une attention particulière à l'origine de la ressource: (i) les pluies, (ii) l'irrigation à partir d'eau de surface - barrages et sources naturelles - ainsi que les eaux souterraines - puits et forages et même des sources non conventionnelles comme le traitement des eaux usées et le dessalement de l'eau de mer, et finalement (iii) l'eau virtuelle qui correspond à la consommation en eau effectuée pour élaborer un produit, ensuite importé dans une localité où il sera consommé. En effet, cette dernière doit être appréhendée finement, car elle représente un moyen commode de s'affranchir du manque d'eau (Antonelli et Tamea, 2014). Par exemple, pour nourrir son bétail l'éleveur peut produire des céréales ou du fourrage mais aussi acheter des aliments concentrés qui sont en grande partie importés. En outre, les scénarios du changement climatique prédisent tous que la vulnérabilité sociale et économique des populations agricoles va s'accentuer au Maroc (Schilling et al., 2020). Ceci sera encore plus ressenti dans les systèmes de production pluviaux qui occupent plus de $80 \%$ de la surface agricole du pays. C'est inquiétant pour les générations futures, d'autant que l'irrigation a toujours été considérée comme l'unique moteur de développement alors qu'elle a révélé des limites évidentes. Ainsi, le PMV a alloué des budgets conséquents à l'extension de l'irrigation localisée ou « goutte à goutte » afin de convertir en une décennie près de 600000 ha, soit le tiers de la surface irriguée. Ceci devait assurer, selon les promoteurs de ce programme, des économies d'eau importantes. Toutefois, des suivis rigoureux révèlent que cette économie d'eau rapportée à l'hectare a été bien moindre que prévue du fait d'un manque de formation des agriculteurs (Benouniche et al., 2014) et qu'en réalité, au niveau national, il a été constaté une extension des surfaces irriguées, par la substitution des cultures pluviales par l'arboriculture, le maraîchage, le maïs, etc. qui ont tous pu bénéficier de l'irrigation localisée. Au final, dans de nombreux bassins hydrauliques, surtout les plus arides, et en l'absence de mécanismes stricts de régulation, les prélèvements d'eau à partir des nappes souterraines ont explosé, entraînant leur épuisement à court ou moyen terme (Molle et Tanouti, 2017).

Les logiques économiques sous-tendant ces choix ont induit une marginalisation des cultures pluviales et notamment des céréales, y compris en zones irriguées, dont les performances constituaient le véritable baromètre du secteur agricole au Maroc. Aujourd'hui encore, la production annuelle de céréales continue pourtant de déterminer une bonne partie de la croissance économique du pays. À l'opposé, l'irrigation a été érigée en modèle emblématique d'une agriculture moderne, octroyant à ceux qui la pratiquent des subventions conséquentes (quel que soit leur captal de départ), une considération sociale certaine, au vu des compétences qu'elle nécessite et de sa contribution à l'effort d'exportation du pays. Cette évolution a débouché sur une spécialisation accrue des systèmes de production en mésestimant les complémentarités entre les différents types de cultures, les ateliers de production (agriculture et élevage), en particulier dans les zones irriguées. En outre, elle a amplifié le mythe du meilleur rendement et de la forte rentabilité des cultures irriguées, sans tenir compte des externalités négatives (pollution des eaux et des sols par les intrants chimiques) et des risques de dégradation des ressources hydriques pouvant entraîner l'effondrement de toute l'agriculture dans les zones les plus arides ... Pire, elle a entraîné au cours de la dernière décennie des surplus de 


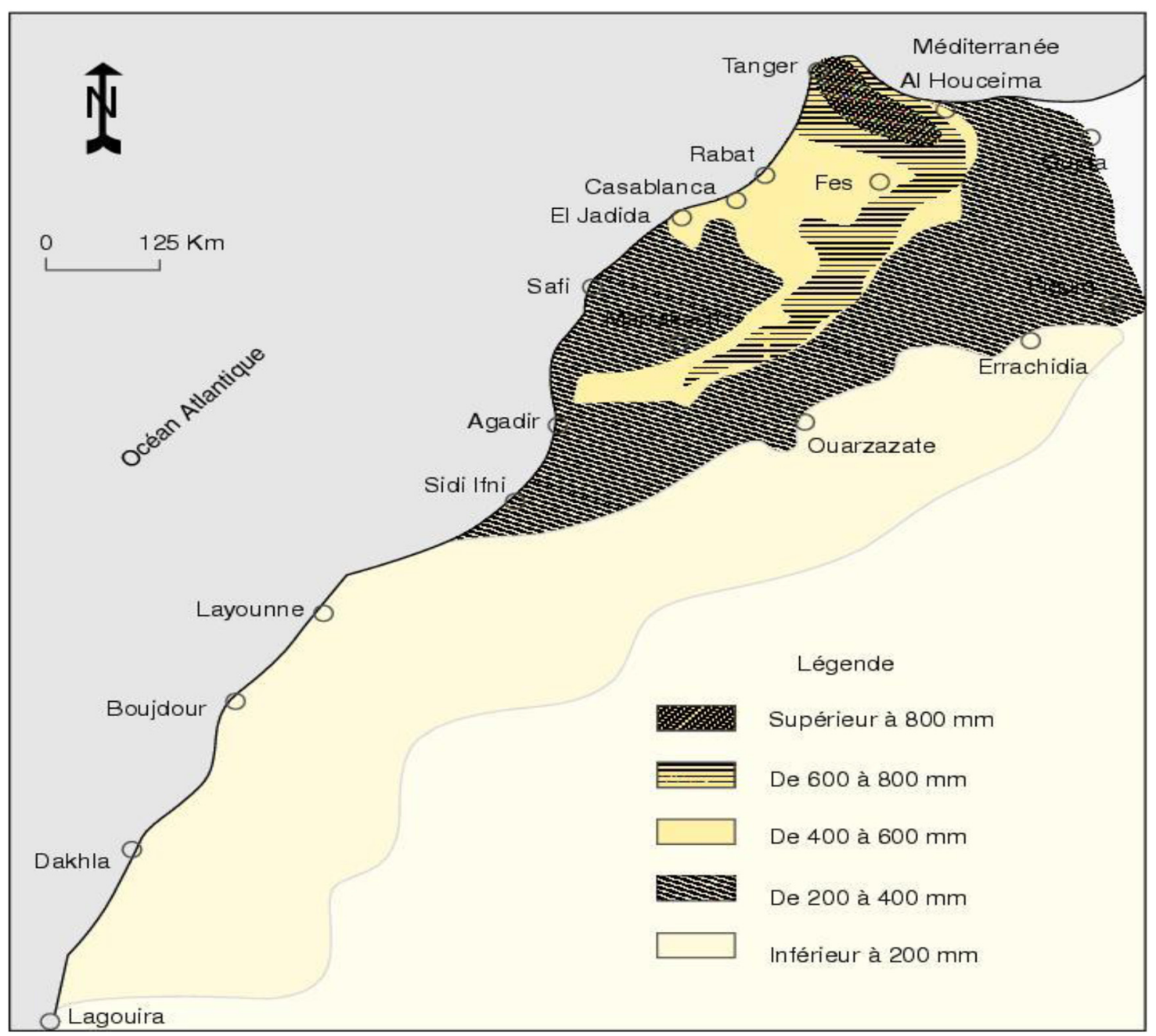

Fig. 1. Niveaux de précipitations annuels moyens au Maroc.

Fig. 1. Average annual levels of rainfall in Morocco.
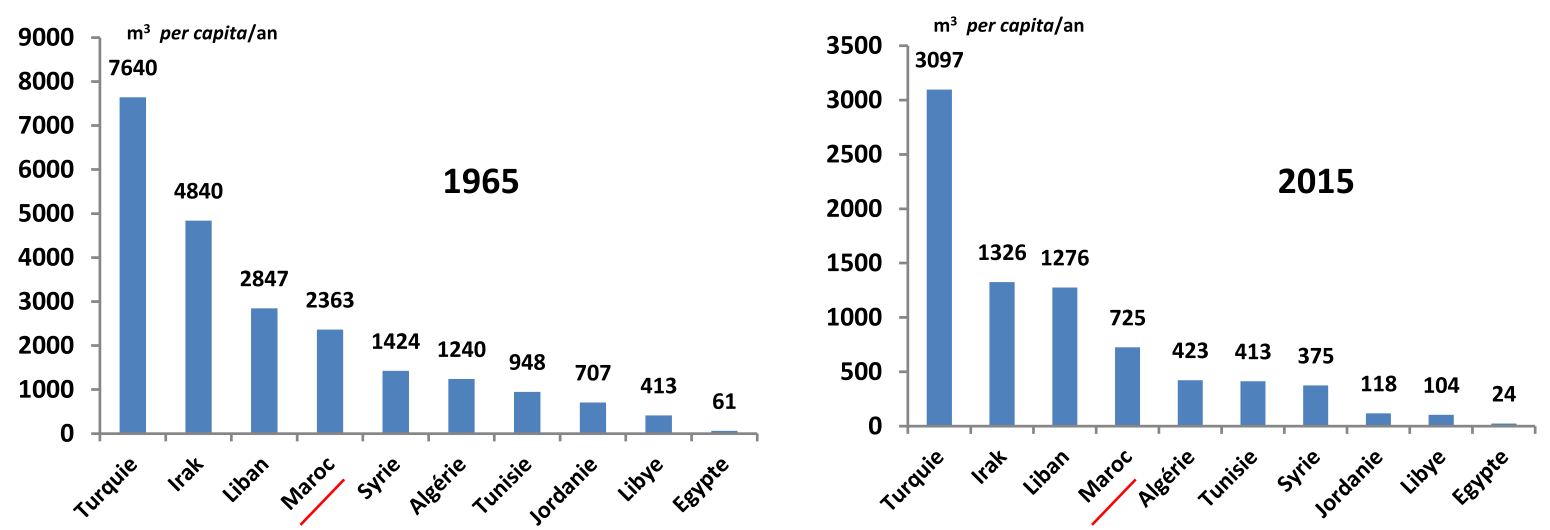

Fig. 2. Volumes annuels d'eau renouvelable per capita dans les pays du Maghreb et du Proche Orient. Source: World Bank (2017).

Fig. 2. Annual renewable volumes of water per capita in the Maghreb and Near East countries.

production de certains fruits et légumes (agrumes, tomates, pêches, etc.), aboutissant au contraire du résultat escompté, qui était une augmentation de la valorisation économique de l'eau $\left(€\right.$ par $\left.\mathrm{m}^{3}\right)$.
De nombreuses observations de terrain confirment ces tendances fâcheuses. Ainsi, des difficultés de commercialisation des agrumes, issues d'un accroissement rapide des quantités produites à partir de plantations subventionnées, 


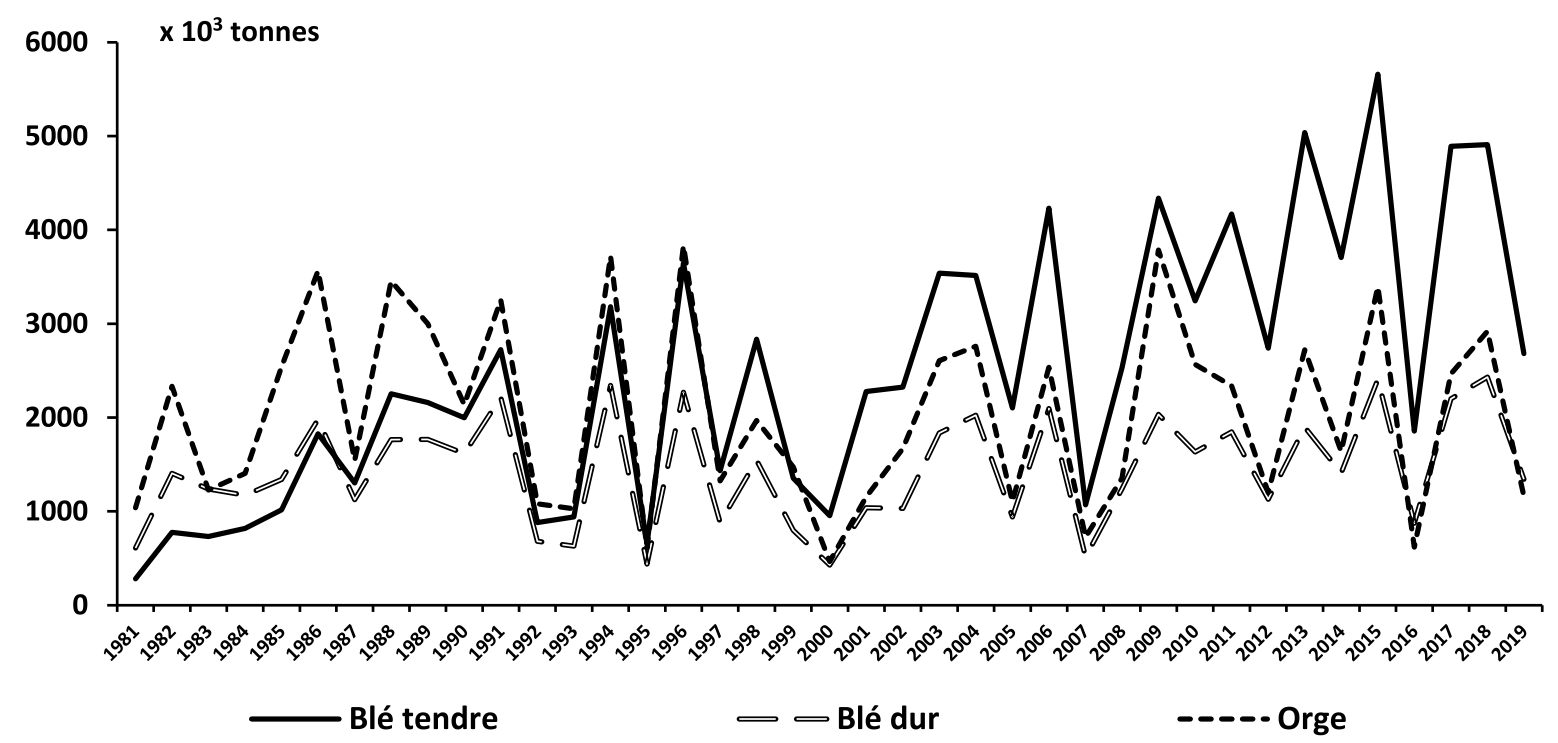

Fig. 3. Variabilité inter-annuelle de la production des principales céréales au Maroc. Source: Adapté de ONICL (2020).

Fig. 3. Inter-annual variability of the output of the main cereals in Morocco.

parfois dans des environnements très arides (moins de $100 \mathrm{~mm}$ de pluie par an, alors que ces cultures nécessitent près de $1000 \mathrm{~mm} /$ an ...), exacerbées par le manque de compétitivité sur les marchés mondiaux (prix, qualité), ont été vivement ressenties et elles ont amené certains agriculteurs à arracher leurs arbres. Ceci a aussi été précipité par l'amplification de la contrainte hydrique. En effet, dans certaines régions comme les périmètres irrigués du Souss-Massa (sud-ouest du pays) et de la Moulouya (nord-est du pays), les autorités qui gèrent les barrages ont alerté les agriculteurs sur l'arrêt du service de l'eau, les volumes stratégiques étant réservés exclusivement aux centres urbains. Une évolution assez similaire a été observée dans les zones oasiennes, où l'extension de la surface irriguée sur des franges désertiques s'est réalisée exclusivement à partir des nappes souterraines. Ce type d'investissements a été orienté vers la culture de la pastèque et de variétés marchandes du palmier dattier, créant une rupture marquée entre l'oasis traditionnelle diversifiée de polyculture-élevage et les extensions à base de monocultures supposées plus «modernes» (Hamamouche et al., 2018). Logiquement, les mêmes phénomènes de surproduction des pastèques et d'épuisement des nappes sont apparus, accentués par le climat très aride de ces régions. Aussi, la stabilité de l'approvisionnement en eau des centres urbains n'y est-elle plus garantie. En outre, la rentabilité de la pastèque est relative, puisque l'offre peut dépasser la demande locale, et le marché d'exportation est volatil. De surcroît, le transport des pastèques, produit pondéreux, est très coûteux, avec une émission en gaz à effet de serre conséquente. Un autre exemple révélateur de cette logique d'intensification poussée par l'irrigation consiste en la substitution des fourrages pluviaux (avoine, orge, etc.) par le maïs ensilé, surtout pour les bovins laitiers, du fait d'un rendement en biomasse élevé. Les promoteurs de ce choix fourrager ne mentionnent cependant ni son impact direct sur les nappes (en tant que culture d'été intégralement irriguée), ni sa teneur très limitée en azote impliquant une dépendance totale vis-à-vis de tourteaux de protéagineux importés, ni les potentiels risques de présence dans le lait de traces de pesticides épandus sur le maïs (Le Faouder et al., 2007).

Tous ces constats n'empêchent pas les producteurs qui ont bénéficié de subventions de solliciter davantage d'aides, sous forme d'un appui au pompage solaire, ce qui va accroître l'usage de l'eau du fait du faible coût de cette technologie et de la possibilité d'accéder à des eaux plus profondes (au-delà de $100 \mathrm{~m}$ ). Mais ceci ne pourra qu'accentuer l'exclusion sociale en milieu rural; ceux qui ne peuvent pas creuser plus profondément par manque de capital étant forcés de se retirer (Ameur et al., 2017). L'extension de la surface irriguée et l'intensification de l'irrigation ont ainsi été érigées en véritable doxa par un grand nombre d'acteurs du secteur agricole, des producteurs aux techniciens et des cadres du développement aux chercheurs, imposant toujours davantage de mobilisation d'eau. Cela inhibe toute réflexion vers d'autres logiques de développement, comme la valorisation en priorité des eaux pluviales et la complémentarité, à l'échelle des territoires, entre les ateliers de production basés sur l'eau pluviale et les cultures irriguées. En effet, la production de céréales du Maroc n'a quasiment pas évolué depuis le lancement du PMV, malgré la promotion d'un système semencier renouvelé (Bishaw et al., 2019). La production demeure largement impactée par les aléas climatiques, impliquant une variabilité marquée (Fig. 3). Le PMV n'a pas induit de changement significatif de pratiques de production, mais il a consolidé la substitution de l'orge par le blé tendre, vu les évolutions des habitudes alimentaires de la population marocaine. L'amélioration des rendements de céréales demeure limitée (une moyenne de 1,64 tonnes par ha durant la période du PMV, alors qu'elle était de 1,17 tonnes par ha entre 2001 et 2007, selon ONICL, 2020), inférieure à la moyenne africaine (2,34 tonnes par ha). Malgré les efforts pour généraliser les usages des engrais dans un pays leader en termes de production et de transformation des phosphates (Lyon et al., 2020), les niveaux limités de productivité des 
céréales reflètent une gestion perfectible de l'eau pluviale et de la fertilité des sols. Ils démontrent aussi les faiblesses structurelles du système de conseil agricole, intimant que des interventions sur le long terme sont nécessaires pour assurer la diffusion des bases de l'agriculture de conservation, comme cela a été démontré dans des contextes similaires (Bahri et al., 2019). La focalisation sur le développement des surfaces irriguées est basée sur la théorie des avantages comparatifs, qui pousse l'agriculture du Maroc à exporter toujours plus de fruits et légumes en contribuant à l'assèchement des nappes à l'instar de ce qui est relevé dans d'autres régions semi-arides du monde (Dalin et al., 2017), tout en mobilisant une main-d'œuvre bon marché. Elle astreint aussi le pays à importer l'essentiel de ses produits vivriers de base (céréales et légumineuses, huiles végétales, sucre, aliments du bétail, etc.). C'est ainsi que pour les 9 premiers mois de l'année 2020, les importations de blé tendre ont crû significativement du fait de la sécheresse et de la volonté des pouvoirs publics de constituer des stocks de sécurité, atteignant un record de 5,9 millions de tonnes. Enfin, pareille stratégie passe sous silence tous les impacts environnementaux qu'elle génère (baisse de la fertilité des sols, épuisement des nappes, pollution des sols et des eaux du fait de l'utilisation croissante et non régulée de pesticides et fertilisants, perte de biodiversité, etc.) et promeut un modèle de développement dont les limites ont été dévoilées par l'irruption de la pandémie: dépendance alimentaire, vulnérabilités par rapport à la volatilité des prix des denrées exportées, manque d'eau (la distribution étant devenue rationnée dans certaines villes et dans de nombreux périmètres agricoles).

\section{Penser un modèle novateur de développement agricole}

La pandémie de la Covid-19 doit être considérée comme une période opportune pour se livrer à un bilan objectif des politiques publiques antérieures ou en cours, dont le PMV, et pour identifier des voies d'évolution durable du secteur agricole. L'intensification des usages d'eau doit être questionnée. La réflexion doit s'accompagner d'une évaluation non seulement des volumes d'eau mobilisés mais aussi de leur origine (Hoekstra et Mekonnen, 2012). En outre, accroître la productivité de l'eau d'irrigation devrait aussi être un objectif pressant, car elle demeure limitée y compris à travers les produits exportés (Schyns et Hoekstra, 2014), l'essentiel des efforts ayant ciblé les techniques d'irrigation sans l'accompagnement nécessaire à la maîtrise des pratiques culturales (Kuper et al., 2009). Les politiques publiques agricoles doivent être révisées en accordant plus d'importance à l'eau pluviale (Rockström et al., 2009). Ceci devrait être la pierre angulaire des choix futurs, en réorientant les programmes d'appuiconseil vers les céréales, les légumineuses et l'élevage adossé aux parcours et aux fourrages non irrigués, dans une logique de généralisation des préceptes de l'agro-écologie, y compris dans les zones irriguées (Ameur et al., 2020). À cet égard, Sraïri et al. (2016) ont démontré que dans les conditions de la plaine du Saïss (avec $500 \mathrm{~mm}$ de pluie par an et une agriculture surtout pluviale qui s'est récemment convertie à l'irrigation à partir d'eaux souterraines), l'élevage bovin était surtout dépendant de l'eau pluviale, mettant en valeur des fourrages tels que l'avoine et le bersim ou trèfle d'Alexandrie et des coproduits de céréales (pailles, chaumes, etc.). Ces résultats impliquent qu'un développement des filières lait et viande rouge n'aggraverait pas la baisse continue des nappes souterraines, lorsqu'elles reposent sur les fourrages produits avec de l'eau pluviale. Ce n'est pas le cas du maraîchage et de l'arboriculture qui se sont développés à vive allure dans cette région, avec les subventions du PMV et sans une maîtrise rigoureuse des quantités d'eau utilisée. De plus, l'élevage est essentiel pour la résilience des exploitations, en produisant le fumier, indispensable à l'entretien de la fertilité des sols, et surtout en diversifiant leurs revenus face à la volatilité des prix de vente des fruits et légumes (Ryschawy et al., 2013).

Le deuxième enseignement issu de la crise sanitaire de la Covid-19 est que la gouvernance des filières concernant des milliers d'agriculteurs doit impérativement reposer sur une approche inclusive et ascendante, où les différentes parties (producteurs, industriels de la transformation, revendeurs, consommateurs et pouvoirs publics) disposent d'espaces de négociation pour débattre des sujets les plus épineux: évaluation de la qualité et paiement adapté, appui technique aux producteurs, distribution équitable de la valeur, etc. (Sraïri et al., 2009; Ourabah Haddad et al., 2017). Cette question de co-gestion des filières n'avait pas été intégrée dans la conception du PMV, pas plus que le renforcement des capacités des organisations des petits producteurs. Une bonne partie des ressources du PMV ont surtout favorisé l'installation de «méga-fermes » et l'extension de leurs activités par le biais d'importantes subventions. Par exemple, dans le secteur de l'élevage laitier, les subventions à ces entreprises ont permis l'importation de génisses, l'installation des salles de traite, l'irrigation du maïs, etc. Mais les exploitations familiales de petite taille qui assurent la plus grande partie de l'approvisionnement en lait du pays ont été peu concernées par ces subventions. Elles ont continué à souffrir des mêmes contraintes : prix de vente du lait réduit par rapport aux méga fermes $(0,3$ vs. $0,38 €$ par litre), contre-performances zootechniques liées à une alimentation insuffisante et déséquilibrée, système de conseil inefficace, etc. (Sraïri et al., 2014). Un déficit généralisé de gouvernance de la filière s'est alors matérialisé et il a débouché sur le boycott des produits laitiers par les consommateurs, au printemps et à l'été 2018. Même si le mouvement ciblait uniquement la société leader du marché, il a finalement perturbé tous les pans de la filière, au vu de la chute significative de la consommation. Selon FAOSTAT (2020), la production annuelle de lait aurait baissé de $25 \%$ entre 2017 et 2018 (Fig. 4), ce qui signifie que les sommes très importantes investies dans ce secteur durant le PMV n'ont eu que peu d'impact, d'autant que la production n'a pas retrouvé depuis les niveaux de 2017. Les contraintes à surmonter pour instaurer une filière laitière durable sont connues et elles sont multidimensionnelles (Sraïri et al., 2019). Elles impliquent une gouvernance équitable, inclusive et transparente et cela concerne toutes les filières et l'ensemble du secteur agricole.

La troisième leçon à tirer de la crise induite par la Covid-19 est liée à la dépendance alimentaire et aux marchés extérieurs. Pour y faire face, de nouvelles formes d'agriculture basées sur le minimum d'usages d'intrants externes, comme les pesticides, les engrais azotés de synthèse, etc. doivent être favorisées. Elles doivent prôner le recyclage intégral de la 


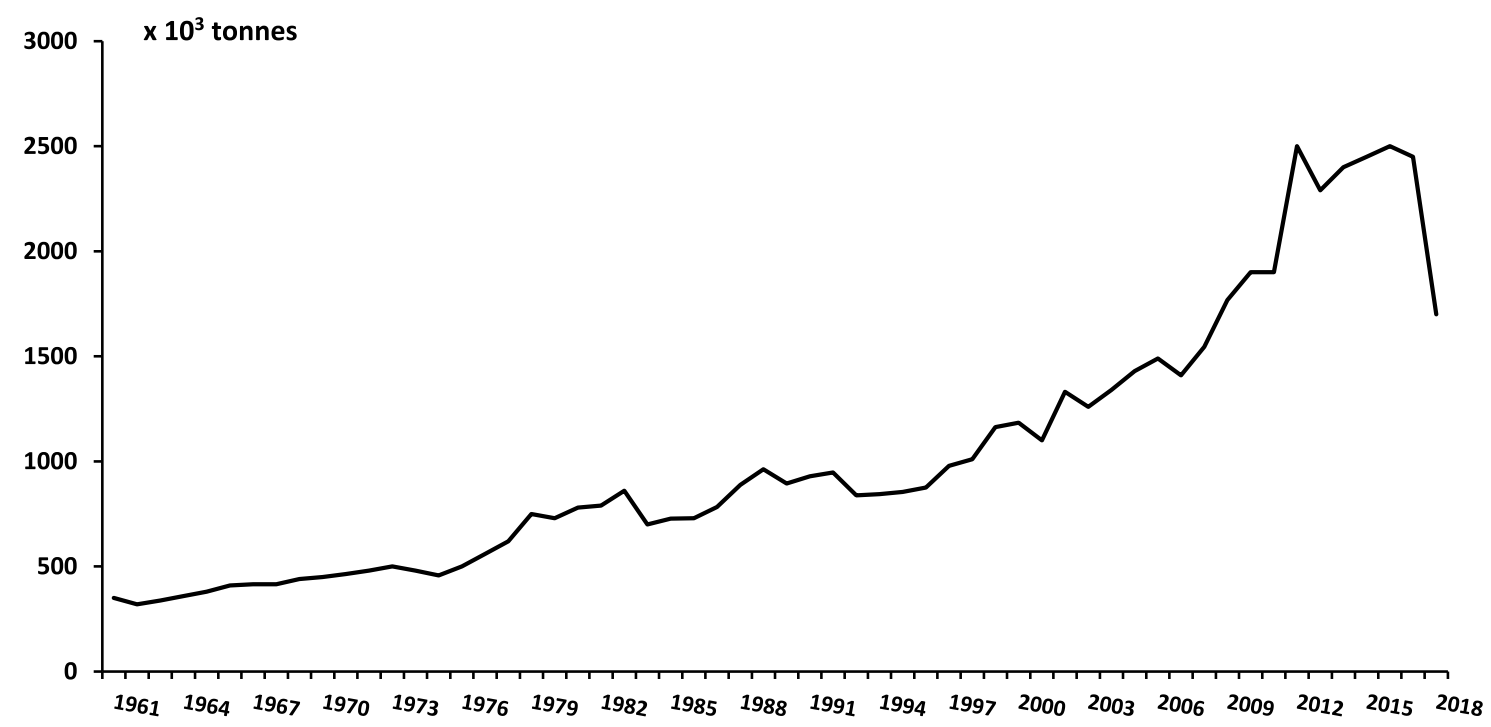

Fig. 4. Évolution de la production annuelle de lait bovin au Maroc (1961-2019). Source: FAOSTAT (2020).

Fig. 4. Evolution of the annual cattle milk output in Morocco.

biomasse et du fumier, dans le respect du principe de l'économie circulaire où l'élevage assure des rôles cruciaux (Peyraud et al., 2019). Ce principe est très éloigné du fonctionnement actuel du secteur avicole marocain, qui a abouti à une autosuffisance en trompe-l'œil en viandes blanches et œufs du pays, alors qu'il est entièrement dépendant d'importations de grains de maïs, de soja et de reproducteurs (Sraïri, 2011). Cela implique aussi une empreinte carbone très élevée pour le transport de ces intrants, sans omettre les risques posés quant à l'émergence de zoonoses (Espinosa et al., 2020).

La pandémie de la Covid-19 a par ailleurs révélé les faiblesses du secteur agricole d'exportation, des contaminations importantes entre ouvriers s'étant déclarées dans des ateliers de conditionnement de fruits rouges, remettant en cause leur meilleure rentabilité par rapport aux productions destinées au marché local. De plus, la baisse des revenus des consommateurs dans les pays importateurs (principalement européens) a aussi contribué à déprécier la demande en produits onéreux, comme les fruits rouges (framboises, fraises, myrtilles, etc.) et les huiles d'argan et d'olive haut de gamme. Aussi, l'émergence de la consommation dans des circuits courts pourrait-elle être une des solutions à promouvoir pour une agriculture plus durable. Mais, l'ensemble de ces orientations sont très éloignées de la philosophie du PMV qui a fait la promotion de la spécialisation, surtout dans de très grandes fermes, caractérisées par l'absence de couplage entre les cultures et l'élevage, et où l'irrigation repose principalement sur la surexploitation des nappes. Ces logiques de production sont aujourd'hui de plus en plus décriées en raison de leurs impacts environnementaux et de leurs vulnérabilités avérées à court et moyen termes (Garrett et al., 2020).

$\mathrm{Au}$ vu des évolutions récentes et en tenant compte de la volatilité des prix des produits agricoles sur les marchés internationaux, il est temps de réhabiliter la notion clé de souveraineté alimentaire, puisque le Maroc continue d'importer près de $200 \mathrm{~kg}$ per capita et par an de céréales (surtout du blé, mais aussi du maïs pour la volaille). Reconnaître les spécificités des secteurs alimentaires et agricoles et en particulier leur importance pour la paix sociale implique de prévoir des investissements conséquents dans les systèmes de production locaux de produits vivriers pour éviter la dépendance aux marchés extérieurs et la vulnérabilité du système alimentaire face à la volatilité des prix. La réflexion approfondie sur la durabilité des systèmes alimentaires doit être couplée à celle portant sur les modèles de production et l'éducation des consommateurs. En effet, dans un pays qui adhérait totalement aux préceptes du régime alimentaire méditerranéen, des modifications rapides des habitudes de consommation ont induit une augmentation du surpoids et de l'obésité : respectivement 46,8 et $16,4 \%$ de la population selon l'OMS (WHO, 2011). En outre, valoriser au maximum l'eau pluviale permet de réduire l'usage d'énergie fossile pour l'exhaure de l'eau, tout en garantissant une contribution importante des denrées vivrières d'origine locale (céréales et légumineuses) dans la diète, un des piliers de systèmes alimentaires durables (Willett et al., 2019). Finalement, promouvoir les circuits courts permettra aussi de limiter les usages d'énergie fossile nécessaire pour l'importation de millions de tonnes d'aliments, pour participer à l'effort mondial de réduction des émissions de gaz à effet de serre.

\section{Conclusion}

La pandémie de la Covid-19 a révélé les contributions cruciales de l'agriculture au quotidien des citoyens, comme l'approvisionnement en denrées alimentaires, l'entretien des territoires, la création de millions d'emplois, etc. Ces fonctions se sont maintenues grâce aux mesures prises par les autorités pour la continuité des activités agricoles durant le confinement, ce qui n'a pas été possible pour des secteurs comme le tourisme ou l'événementiel. La pandémie a toutefois dévoilé les faiblesses structurelles du secteur agricole du Maroc, les citoyens ayant pris conscience des quantités énormes de produits vivriers importés. Cela correspond à une dépense 
annuelle de près de $110 €$ per capita, qui doit être considérée comme un apport d'eau virtuelle, permettant aussi de soulager quelque peu le stress hydrique dont souffre le pays. Ces importations ont pu se faire à un prix relativement abordable, $y$ compris pour les familles ayant perdu leurs emplois et ayant de ce fait bénéficié de subsides publics. Toutefois que serait-il advenu si les prix des produits alimentaires avaient augmenté soudainement sur les marchés mondiaux, comme durant la crise de 2007/2008 ? De plus, ces importations bon marché de céréales et de sucre n'accélèrent-elles pas les changements d'habitudes alimentaires et l'explosion des cas d'obésité ?

La pandémie s'étant déclarée dans une année très sèche, ses effets ont été plus aigus pour les revenus des agriculteurs et seront ressentis sur le long terme. Elle a notamment dévoilé les insuffisances des politiques adoptées pour le secteur agricole, en particulier de choix de modèles de production, et d'options libérales favorisant l'exportation de fruits et de légumes primeurs dont les prix sont très volatils sur les marchés internationaux, et l'importation massive de produits vivriers de base. Ces faiblesses doivent être finement analysées et leurs effets et impacts quantifiés afin d'éviter le retour des vieux réflexes qui considèrent l'expansion de l'irrigation comme l'unique voie de développement agricole du pays. Au contraire, la pandémie doit être considérée comme une opportunité pour ouvrir un débat responsable et serein sur les usages de l'eau, surtout d'irrigation à partir de sources non renouvelables, quitte à réviser les ambitions, tout en convenant de l'urgence de recourir aux connaissances scientifiques avérées pour concevoir les choix du futur, à l'instar de ce qui a été fait pour gérer la dissémination du virus.

Au final, tout cela impose un changement de paradigme en préparant les acteurs du secteur agricole à accepter d'allouer plus de moyens à la valorisation d'une diversité de ressources naturelles, et en premier l'eau pluviale. Cela devra se faire sur des bases scientifiques et par la promotion de bonnes pratiques, ce qui implique d'accorder un intérêt particulier à la formation, dans ses différents niveaux (agriculteurs, techniciens, ingénieurs, etc.). Ceci constitue une condition sine qua non pour concrétiser les objectifs de la future stratégie agricole gouvernementale, dénommée "Green Generation», avec une croissance inclusive, qui attire les jeunes actifs et garantisse une rémunération décente pour tous les acteurs du secteur agricole. Enfin, il convient de voir les réalités en face, et de considérer qu'au vu des contraintes structurelles dont souffre le pays (manque d'eau, foncier sécurisé exigu, fertilité des sols limitée, etc.) il est illusoire de continuer à magnifier le mythe de sa puissance agricole, destinée à être la locomotive de l'économie.

Remerciements. L'auteur remercie les agriculteurs de différentes régions du Maroc pour lui avoir permis de mener à bien le suivi de leur exploitation depuis plusieurs années et ainsi, de saisir la complexité des systèmes qu'ils gèrent. Il leur est redevable d'avoir répondu à ses questions relatives à l'évolution de leurs activités suite à l'émergence soudaine de la pandémie de la Covid-19.

\section{Références}

Acuto M. 2020. Covid-19: Lessons for an urban(izing) world. One Earth 2: 317-319. https://doi.org/10.1016/j.oneear.2020.04.004.
Akesbi N. 2015. Qui fait la politique agricole au Maroc? Ou quand l'expert se substitue au chercheur... Annales de l'INRA Tunisie 88: 104-126.

Ameur F, Kuper M, Lejars C, Dugué P. 2017. Prosper, survive or exit: contrasted fortunes of farmers in the groundwater economy in the Saiss plain (Morocco). Agricultural Water Management 191: 2072017.

Ameur F, Amichi H, Leauthaud C. 2020. Agroecology in North African irrigated plains? Mapping promising practices and characterizing farmers' underlying logics. Regional Environmental Change 20: 133. https://doi.org/10.1007/s10113-020-01719-1.

Antonelli M, Tamea S. 2014. Food-water security and virtual water trade in the Middle East and North Africa. International Journal of Water Resources Development 31: 326-342. https://doi.org/ 10.1080/07900627.2015.1030496.

Bahri H, Annabi M, M'hamed HC, Frija A. 2019. Assessing the longterm impact of conservation agriculture on wheat-based systems in Tunisia using APSIM simulations under a climate change context. Science of the Total Environment 692: 1223-1233.

Barakate F, Handoufe A. 1998. Approche agroclimatique de la sécheresse agricole au Maroc. Sécheresse 9: 201-210.

Benouniche M, Kuper M, Hammani A, Boesveld H. 2014. Making the user visible: analysing irrigation practices and farmers' logic to explain actual drip irrigation performance. Irrigation Science 32: 405-420.

Bishaw Z., Yigezu YA, Niane A, Telleria RJ, Najjar D, (eds). 2019. Political economy of the wheat sector in Morocco: seed systems, varietal adoption, and impacts. Beirut, Lebanon: International Center for Agricultural Research in the Dry Areas, $300 \mathrm{p}$.

Bossenbroek L, Van der Ploeg JD, Zwarteveen M. 2015. Broken dreams? Youth experiences of agrarian change in Morocco's Saïss region. Cahiers Agricultures 24(6): 342-348. DOI: 10.1684/ agr.2015.0776.

Cournut S, Chauvat S, Correa P, Dos Santos Filho JC, Dieguez F, Hostiou N, et al. 2018. Analyzing work organization by the Work Assessment Method: a meta-analysis. Agronomy for Sustainable Development 38: 58. https://doi.org/10.1007/s13593-018-0534-2.

Dalin C, Wada Y, Kastner T, Puma MJ. 2017. Groundwater depletion embedded in international food trade. Nature 543: 700-704. https:// doi.org/10.1038/nature21403.

Espinosa R, Tago D, Treich N. 2020. Infectious diseases and meat production. Environmental and Resource Economics 76: 10191044. https://doi.org/10.1007/s10640-020-00484-3.

Espita A, Rocha N, Ruta M. 2020. Covid-19 and food protectionism: The impact of the pandemic and export restrictions on world food markets. World Bank Policy Research Working Paper No. 9253, 30 p. https://papers.ssrn.com/sol3/papers.cfm?abstract_id= 3605887.

FAOSTAT. 2020. Production annuelle de lait bovin au Maroc (19612018). Retrouvé sur: http://www.fao.org/faostat/fr/\#data/QL.

Faysse N. 2015. The rationale of the Green Morocco Plan: missing links between goals and implementation. Journal of North African Studies 20: 622-634. https://doi.org/10.1080/ 13629387.2015 .1053112 .

Garrett RD, Ryschawy J, Bell LW, Cortner O, Ferreira J, Garik AVN, et al. 2020. Drivers of decoupling and recoupling of crop and livestock systems at farm and territorial scales. Ecology and Society 25: 24. https://doi.org/10.5751/ES-11412-250124.

Graeber D. 2018. Bullshit Jobs. Paris: Editions Les Liens qui Libèrent, $416 \mathrm{p}$.

Hamamouche MF, Kuper M, Amichi H, Lejars C, Ghodbani T. 2018. New reading of Saharan agricultural transformation: Continuities of ancient oases and their extension. World Development 107: 210-223. 
Haut Commissariat au Plan. 2015. Enquête nationale sur la consommation et la dépense des ménages au Maroc. http:// www.hcp.ma/Introduction-de-Monsieur-Ahmed-LAHLIMIALAMI-Haut-Commissaire-au-Plan-a-la-presentation-des-resul tats-de-L-enquete_a1819.html.

Hoekstra AY, Mekonnen MM. 2012. The water footprint of humanity. Proceedings of the National Academy of Science (PNAS) 109: 3232-3237. https://doi.org/10.1073/pnas.1109936109.

Kuper M, Bouarfa S, Errahj M, Faysse N, Hammani A, Hartani S. 2009. A crop needs more than a drop: towards a new praxis in irrigation management in North Africa. Irrigation and Drainage 58: S231-S239.

Laborde D, Martin W, Swinnen J, Vos R. 2020. Covid-19 risks to global food security. Science 369: 500-502.

Le Faouder J, Bichon E, Brunschwig P, Landelle R, Andre F, Le Bizec B. 2007. Transfer assessment of fipronil residue from feed to cow milk. Talanta 73: 710-717.

Leveau R. 1972. Le fellah marocain, défenseur du trône. Paris : Editions la Découverte, $275 \mathrm{p}$.

Lyon C, Cordell D, Jacobs B, Martin-Ortega J, Marshall R, CamargoValero MA, et al. 2020. Five pillars for stakeholder analyses in sustainability transformations: The global case of phosphorus. Environmental Science \& Policy 107: 80-89.

Mahdi M. 2014. Devenir du foncier agricole au Maroc : un cas d'accaparement des terres. New Medit 13: 2-10.

Molle F, Tanouti O. 2017. Squaring the circle: Agriculture intensification vs. water conservation in Morocco. Agricultural Water Management 192: 170-179. https://doi.org/10.1016/j. agwat.2017.07.009.

Office des Changes. 2020. Résultats des échanges extérieurs à décembre 2019. Retrouvé sur: www.oc.gov.ma/fr/actualites/ communique-resultats-des-echanges-exterieurs-a-fin-decembre2019.

ONICL (Office National Interprofessionnel des Céréales et des Légumineuses). 2020. Production des principales cultures céréalières (blé dur et tendre, orgeet maïs). Retrouvé sur: www.onicl. org.ma.

Ourabah Haddad N, Ton G, Sraïri MT, Bijman J. 2017. Organisational challenges of Moroccan dairy cooperatives and the institutional environment. International Journal on Food Systems Dynamics 8: 236-249. http://centmapress.ilb.uni-bonn.de/ojs/index.php/fsd/arti cle/view/835/720.

Peyraud J-L, Aubin J, Barbier M, Baumont R, Berri C, Bidanel J-P, et al. 2019. Science for tomorrow's livestock farming: A forward thinking conducted at INRA. INRA Productions Animales 32: 323338 . https://doi.org/10.20870/productions-anima les.2019.32.2.2591.

Rockström J, Falkenmark M, Karlberg L, Hoff H, Rost S, Gerten D. 2009. Future water availability for global food production: The potential of green water for increasing resilience to global change. Water Resources Research 45: W00A12.

Ryschawy J, Choisis N, Choisis JP, Gibon A. 2013. Paths to last in mixed crop-livestock farming: lessons from an assessment of farm trajectories of change. Animal 7: 673-681.
Sager F, Mavrot C, Hinterleitner M, Kaufmann D, Grosjean M, Stocker TF. 2020. A six-point checklist for utilization-focused scientific policy advice. Climate Policy In press. https://doi.org/ 10.1080/14693062.2020.1757399.

Schilling J, Hertig E, Tramblay Y, Scheffran J. 2020. Climate change vulnerability, water resources and social implication in North Africa. Regional Environmental Change 20: 15. https://doi.org/ 10.1007/s10113-020-01597-7.

Schyns JF, Hoekstra AY. 2014. The added value of water footprint assessment for national water policy: a case study for Morocco. PLOS One. https://doi.org/10.1371/journal.pone.0099705.

Sraïri MT. 2011. Le développement de l'élevage au Maroc : succès relatifs, dépendance alimentaire. Le Courrier de l'Environnement de l'INRA 60: 91-101. http://www7.inra.fr/dpenv/pdf/C60TaherS rairi.pdf.

Sraïri MT, Benhouda H, Kuper M, Le Gal P-Y. 2009. Effect of cattle management practices on raw milk quality on farms in a two stage dairy chain. Tropical Animal Health and Production 41: 259-272.

Sraïri MT, Chergui S, Igueld H, Sannito Y. 2014. Performances des exploitations laitières familiales au Maroc: arguments pour l'amélioration du prix du lait à la ferme et de l'appui technique. Revue d'Elevage et de Médecine vétérinaire des Pays tropicaux 67: 183-191. http://remvt.cirad.fr/cd/derniers_num/2014/ REMVT14_183_191.pdf.

Sraïri MT, Benjelloun R, Karrou M, Ates S, Kuper M. 2016. Biophysical and economic water productivity of dual purpose cattle farming. Animal 10: 283-291. http://journals.cambridge.org/ action/displayAbstract fromPage $=$ online $\&$ aid $=10082209 \&$ full textType $=$ RA\&fileId $=$ S1751731115002360.

Sraïri MT, Bahri S, Ghabiyel Y. 2018. Work management as a means to adapt to constraints in farming systems: a case study from two regions in Morocco. Cahiers Agricultures 27(1): 15007. DOI: 10.1051/cagri/2017066.

Sraïri MT, Chatellier V, Corniaux C, Faye B, Aubron C, Hostiou N, et al. 2019. Durabilité du développement laitier : réflexions autour de quelques cas dans différentes parties du monde. INRA Productions Animales 32: 339-358. https://doi.org/10.20870/ productions-animales.2019.32.3.2561.

World Bank. 2017. Beyond scarcity: Water security in the Middle East and North Africa. MENA Development Report. Washington: The World Bank Group. Available from https://www.worldbank.org/en/ topic/water/publication/beyond-scarcity-water-security-in-themiddle-east-and-north-africa.

World Bank. 2020. Morocco Economic Monitor. July 2020. 44 p. Available from https://documents.worldbank.org/en/publication/ documents-reports/documentdetail.

World Health Organization (WHO). 2011. Non communicable diseases country profiles 2011. Global Report. Available from http://www. who.int/nmh/publications/ncd-profiles2011/en/index.html.

Willett W, Rockström J, Loken B, Springmann M, Lang T, Vermeulen S, et al. 2019. Food in the Anthropocene: the EAT-Lancet commission on healthy diets from sustainable food systems. The Lancet 393: 447-492. https://doi.org/10.1016/S0140-6736(18) 31788-4.

Citation de l'article : Sraïri MT. 2021. Repenser le modèle de développement agricole du Maroc pour l'ère post Covid-19. Cah. Agric. 30: 17. 